\title{
Utility and efficacy of advanced imaging in thoracic disorders
}

\author{
Ammar Chaudhry \\ Department of Diagnostic and Interventional Radiology, City of Hope National Medical Center, Duarte, CA, USA \\ Correspondence to: Dr. Ammar Chaudhry. 1500 E. Duarte Rd., Duarte, CA 91010, USA. Email: achaudhry@coh.org.
}

Submitted Apr 01, 2020. Accepted for publication Apr 20, 2020.

doi: 10.21037/jtd-2019-pitd-11

View this article at: http://dx.doi.org/10.21037/jtd-2019-pitd-11

There have been dramatic improvements in biomedical imaging paving way for real-time noninvasive assessment of disease. Advances in biomedical imaging have led to: (I) more accurate initial disease staging; (II) improvement in medical and surgical treatment selection; and (III) identifying response assessment. For example, development of new diagnostic modalities (e.g., PET-MRI) have allowed for simultaneous acquisition of structural and functional imaging which are providing new novel ways of noninvasively identifying pathologic structural abnormalities and associated functional deficits. Modalities like PET-MRI can more precisely diagnose multifocal diseases like lymphoma or sarcoidosis that can have intrathoracic and extra-thoracic manifestations, but also detect functional cardiac and/or neurologic deficits with improved accuracy; all on one modality.

This special issues features contributions from renowned experts in the field and aims to highlight key developments in the imaging sciences and their respective impact across spectrum of thoracic disorders. The details and application of modern imaging techniques are presented in context of patient management with overall goal of improving readers understanding of the role advanced imaging plays in managing thoracic disorders. This issue also explores future directions of imaging development and its associated impact on patient care.

Bonjoc et al. highlight improvements in the field of thyroid imaging and its impact on patient care. Dr. Hazelton and colleagues review role of advanced imaging in lung transplant pre- and post-treatment evaluation. Dr. Lall et al. highlight clinical utility of precision imaging in esophageal cancer and how advanced in imaging can help distinguish esophageal cancer from non-neoplastic disorders. Dr. Gul and colleagues provide in-depth review of precision imaging and its role in improved diagnostic specificity across spectrum of rheumatologic disorders.

The re-emergence of Theranostics is complimenting development of PET-MRI and older modalities through creation of targeted bifunctional agents that can simultaneously detect disease as well as treat it. Theranostics can provide noninvasive imaging biomarkers that can be of predictive and prognostic value. In this issue, Massarelli and colleagues highlight the importance of immunotherapy in changing survival of patients with thoracic malignancies (e.g., non-small cell lung cancer, small cell lung cancer, etc.) as well as the potential benefit of Theranostics in adding diagnostic specificity. There are currently at least 8 early phase (phase I and phase II) Theranostics studies evaluating safety and efficacy of anti-PD1 and anti-PDL1 imaging agents. Such studies will not only facilitate optimizing patient selection for FDA approved agents such as pembrolizumab, nivolumab, darvulamab, etc. but it can also help inform future therapeutic trials. For example, in this special issue of Salgia and colleagues demonstrate potential role immunotherapy can play in KRAS-mutant non-small cell lung cancer survival. Combining Theranostics studies with therapeutic studies can pave way for precision medicine through non-invasively detecting tumor and explicating key pathways at play in the tumor microenvironment, which can inform optimal patient-specific therapeutic approach. Also, Dr. Chaudhry and colleagues discuss other Theranostics agents (e.g., MIBG for small cell lung cancer, FET for non-small cell lung cancer, gallium PSMA for metastatic neoplasms, etc.).

Rapid advances in Machine learning (ML) and Artificial Intelligence (AI) algorithms are revolutionizing the field of radiology by: (I) accelerating image acquisition; (II) improving image post-processing; (III) increasing diagnostic accuracy. In the last 5 years, over 11,758 articles were 
published (as indexed on PubMed) of which 4,553 articles alone were published in 2019. AI and ML research in thoracic disease is impacting evaluation of rheumatologic disorders, cardiovascular disease, oncology, along with other thoracic disorders. In this issue, Dr. Lin and colleagues from Johns Hopkins show how AI can improve diagnostic accuracy of tuberculosis on chest radiographs alone. In this issue, Dr. Wong reviews strategies that can be used to develop an AI pipeline which can improve diagnosis and patient management across spectrum of disease and improve quality of care. Additionally, Dr. Wong highlights the potential of combining AI with radiomics can help noninvasively characterize thoracic lesions which can add diagnostic specificity beyond what has been achieved in the past.

Advanced thoracic imaging is a unique resource that is integral to advancing patients care and research. This special issue of $7 T D$ is intended to educate and assist physicians and other healthcare providers in their management of patients with broad spectrum of thoracic disorders. Specifically, this issue will be informative for pulmonary and critical care providers, cardiothoracic surgeons, rheumatologists, radiologists, residents and medical students.

We thank all the authors, their staff and institutions for their contribution towards this special issue of fournal of Thoracic Disorder.

\section{Acknowledgments}

I would like to take this opportunity to thank the Editorial Board of Fournal of Thoracic Disease for this unique opportunity. Also, I would like to thank the staff of Fournal of Thoracic Disease for the endless support in organizing this issue.

Funding: None.

\section{Footnote}

Provenance and Peer Review: This article was commissioned by the editorial office, Fournal of Thoracic Disease for the series "Role of Precision Imaging in Thoracic Disease". The article did not undergo external peer review.

Conflicts of Interest: The author has completed the ICMJE uniform disclosure form (available at: http://dx.doi. org/10.21037/jtd-2019-pitd-11). The series "Role of Precision Imaging in Thoracic Disease" was commissioned by the editorial office without any funding or sponsorship. AC served as the unpaid Guest Editor of the series.

Ethical Statement: The author is accountable for all aspects of the work in ensuring that questions related to the accuracy or integrity of any part of the work are appropriately investigated and resolved.

Open Access Statement: This is an Open Access article distributed in accordance with the Creative Commons Attribution-NonCommercial-NoDerivs 4.0 International License (CC BY-NC-ND 4.0), which permits the noncommercial replication and distribution of the article with the strict proviso that no changes or edits are made and the original work is properly cited (including links to both the formal publication through the relevant DOI and the license). See: https://creativecommons.org/licenses/by-nc-nd/4.0/.
Cite this article as: Chaudhry A. Utility and efficacy of advanced imaging in thoracic disorders. J Thorac Dis 2020;12(9):5060-5061. doi: 10.21037/jtd-2019-pitd-11 